\title{
Study of the Healing Effect of Communication with Plants Applying Sensing of Environmental Information Around the Plants
}

\author{
Hidetaka Nambo ${ }^{*}$, Taiki Nakagawa ${ }^{1}$, Haruo Maegawa ${ }^{1}$ and Haruhiko Kimura \\ Division of Electrical Engineering and Computer Science, \\ Graduate School of Natural Science and Technology, Kanazawa University, \\ Kakuma-machi, Kanazawa, Ishikawa 920-1192, Japan \\ 'Division of Electrical and Computer Engineering, \\ Graduate School of Natural Science and Technology, Kanazawa University, \\ Kakuma-machi, Kanazawa, Ishikawa 920-1192, Japan
}

(Received September 28, 2009; accepted March 16, 2010)

Key words: sensor application, bioelectric potential, communication, healing effect

Nowadays, owing to drastic changes in social conditions, people are increasingly becoming stressed. Consequently, the number of people who want to be healed is also increasing. In this study, we focused on the use of plants to reduce stress. We develop a pseudo-communication system with plants using 7 sensors (for temperature, humidity, $\mathrm{CO}_{2}$, and so on), a microphone, and a display. The system will have healing effects such as those of counselors or animal-assisted therapy. Then, a communication experiment is carried out to confirm that the proposed system can reduce user's stress. As a result, from amylase activity, it is found that user stress is reduced when the user uses the system when stressed. Therefore, it is clear that the proposed communication system has some healing effect on stressed users.

\section{Introduction}

Nowadays, owing to drastic changes in social conditions, people are increasingly becoming stressed. Consequently, the number of people who want to be healed is also increasing. The causes of stress are called stressors. Stressors are categorized into 4 types as follows:(1) (1) physical stressors, (2) chemical stressors, (3) biological stressors, and (4) mental stressors. These stressors cause stress in humans, as well as diseases such as schizophrenia or psychosomatic diseases, and adversely affect people's daily life or works.

To reduce the effects of these stresses, many methods have been applied. For example, it is said that counseling is effective. Also, there are many reports on animalassisted therapies or animal-assisted activities.

*Corresponding author: e-mail: nambo@blitz.ec.t.kanazawa-u.ac.jp 
In this paper, we focused on plants to reduce stresses. It is well known that people can be healed by the appearance or flavors of plants. Moreover, plants have many kinds of effects, and these effects also reduce stresses directly or indirectly.

However, the causes of stress vary; therefore, the use of plants only is not enough to reduce all stresses. In this study, we use multisensors such as those for temperature, humidity, and $\mathrm{CO}_{2}$, to develop a pseudo-communication system with plants. The system will provide plants healing effects such as those of counseling or animal-assisted therapy.

In $\S 2$, stressors and the features of a plant are described. In $\S 3$, the purpose of this study is described. In $\S 4$, the outline of the communication system is described. In $\S 5$, an experiment to evaluate the healing effect of the proposed system and considerations are described. Finally, in $\S 6$, our conclusions are provided.

\section{Stressors and Features of Plants}

It is known that plants have many features. In this section, we explain that these features are effective against the stressors described in $\S 1$.

(1) Physical stressors

These stressors arise from an unsuitable environment, such as noises or uncomfortable temperature. To reduce stress due to these stressors, plants are employed. For example, a wall of green leaves or roots functions as a soundproofing material or reduces heat.

(2) Chemical stressors

These stressors arise from odorous substances or air pollutants. For these stressors, it has been reported that plants have an air-purifying function. ${ }^{(2)}$

(3) Biological stressors

These stressors cause diseases, for example, bacteria. These stressors can be reduced by plants, because some plants have germicidal effects. ${ }^{(3)}$

(4) Mental stressors

These stressors arise from troubles about personal relations, mental anxieties or strains. For these stressors, it is said that the appearance or scents of flowers are effective to reduce stress due to the stressor.

As aforesaid, plants are useful for reducing stress. However, it is difficult for mental stress to be reduced by only plants owing to the fact that the cause of mental stress is psychological and is often uncertain. Generally, counseling is utilized to deal with mental stress.

\section{Purpose of Study}

In this paper, we develop a system to deal with the mental stressors described in $\S 2$. Originally, psychotherapy is applied to deal with mental stressors by a medical specialist of the field or by counseling by a counselor. Alternatively, animal-assisted therapy has been applied as a supplementary method of psychotherapy. Furthermore, robot-assisted therapy, which is a substitute for animal-assisted therapy, has been the focus of many studies and expected now, because robots have an advantage over animals from the point 
of sanitation or safety.

In this study, we developed a system to reduce mental stress using plants. Without our system, it is possible to reduce stress owing to the features that plants originally possess. However, it is not enough. As described in $\S 2$, "communication" is required to deal with mental stressors effectively. Therefore, in this paper, we propose a system for communication with a plant using multisensors. By applying both our system and the original features of plants, it will be possible to deal with stress more effectively.

To realize communication with plants, we need to develop a pseudo-emotion model similar to a pet robot model. In this study, the emotion of a plant is generated from environmental conditions such as temperature and humidity, which affect plant growth. Furthermore, bioelectric potential is used as the tactile sensation of a plant. ${ }^{(4)}$

Furthermore, a facial picture of a plant is generated from its emotion. Then, the image and speech recognition are used to communicate with the plant. Using the facial picture will reduce the feeling of wrongness and enable nonverbal communication such as a back-channel feedback or a nod.

\section{Communication System}

In this section, we describe our proposed system.

\subsection{Components of proposed system}

Figure 1 shows the components of the system. The proposed system consists of multisensors for measuring environmental parameters of a plant, a sensor for measuring bioelectric potential, a knowledge base for generating responses of the plant, and communication devices such as a microphone, a speaker, and a display.

The system measures environmental parameters that affect the growth of the plant.

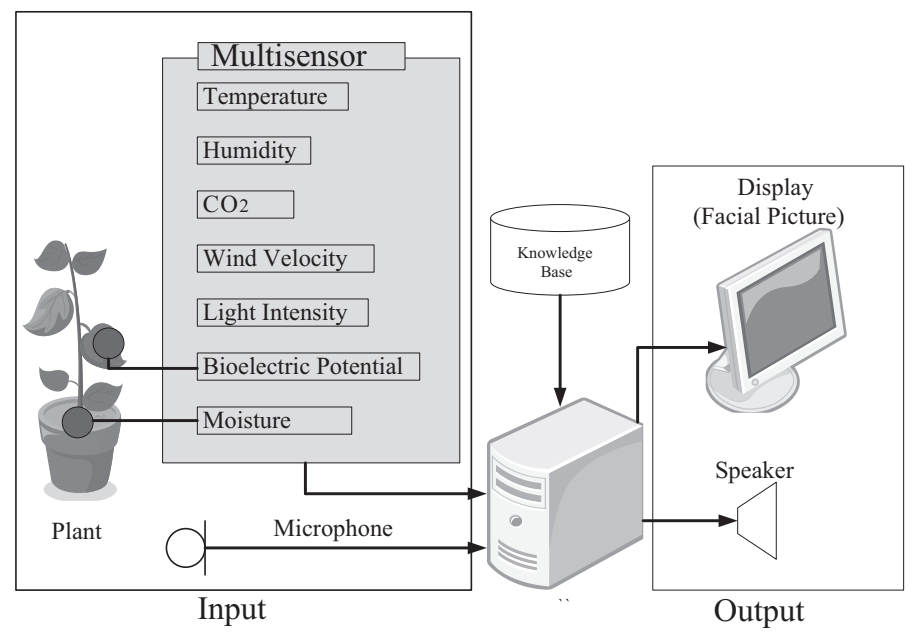

Fig. 1. Components of communication system. 
In this study, temperature, humidity, $\mathrm{CO}_{2}$ density, light quantum, wind velocity, and soil moisture are measured. Sensors for measuring these parameters are shown in Table 1. Furthermore, bioelectric potential is also measured to detect the contact between a user and the plant.

An emotion of the plant is generated from the values acquired by sensors. Simultaneously, a facial picture of the plant is generated. Details are described in $\S 4.2$ and $\S 4.3$. The generated picture is displayed in a system's monitor. Users can communicate with the plant through the picture, microphone, and speaker. The system's responses are generated from the user's question, values obtained by the sensors, and the knowledge base. The flow of communication is shown in Fig. 2, and a picture of the system is shown in Fig. 3.

Table 1

Sensors used to measure environment of the plant.

\begin{tabular}{lc}
\hline Target & Maker/Model \\
\hline Temperature & Comaide/USBT-205 \\
Humidity & \\
$\mathrm{CO}_{2}$ & KANOMAX/CLIMOMASTER MODEL6542 \\
Wind velocity & TandD/PHR-51 \\
Light quantum & Decagon Devices Inc./EM5B \\
Soil moisture & \\
\hline
\end{tabular}

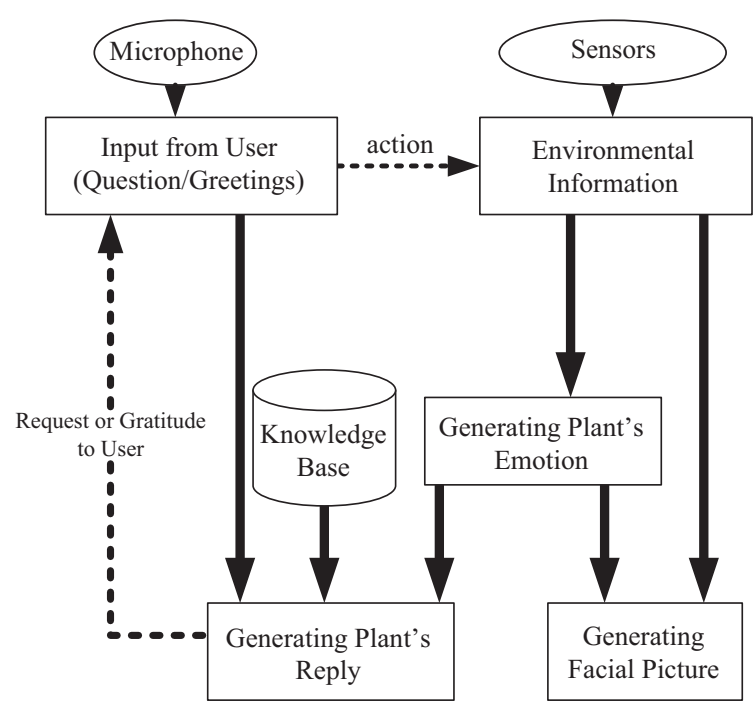

Fig. 2. Communication flow. 


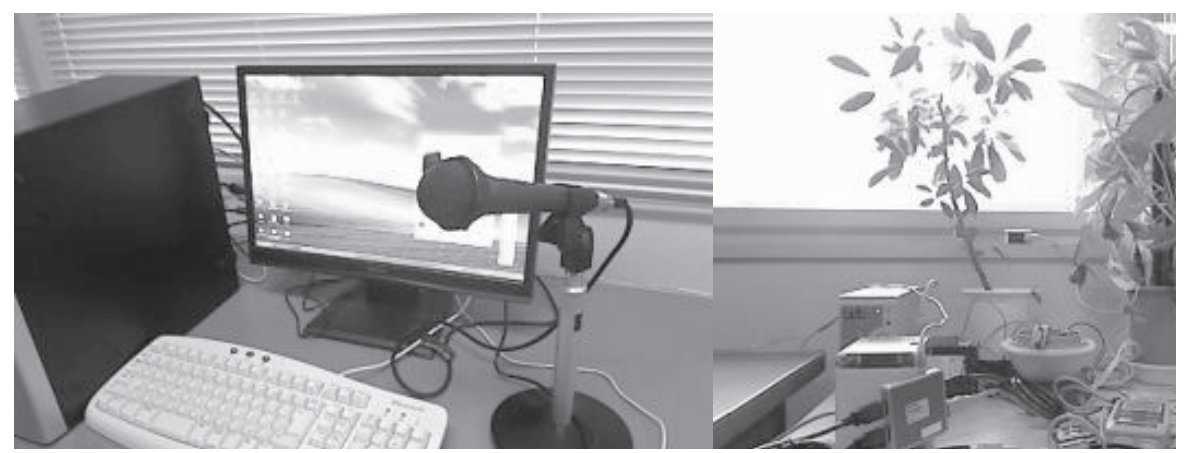

(a)

(b)

Fig. 3. Pictures of communication system. (a) Microphone and display, (b) sensors and plant.

\subsection{Emotion model}

To obtain natural responses from a plant, the plant must have an emotion similar to the emotion of an animal pet when users communicate with the plant. We implemented a simple emotion model for the proposed system.

The emotion model in our system has two emotional conditions, "happy" and "sad." In the model, the plant feels "happy" when it is under good conditions, and it feels "sad" when it is under bad conditions. In this paper, a condition means an environment of the plant that affects its growth. That is, when the environment of the plant is good, the plant feels happy, and when the environment of the plant is not good, the plant feels sad.

In the proposed system, sensors except the bioelectric potential sensor are used to determine the environment of the plant. Then, the emotion of the plant is generated. That is, suitable domains for each parameter are defined beforehand.

\subsection{Facial picture}

In the proposed system, a facial picture is generated and it is used as an interface between a user and the plant when communicating with the plant. The facial picture is generated from environmental parameters of the plant, as well as the emotion. That is, parts of the face in the picture such as the eyes, brows, mouth, and so on correspond to parameters of the environment of the plant. Then, the shapes or colors of parts are transformed on the basis of the values of parameters. Furthermore, the shapes and colors are adjusted to generate the most balanced facial picture when all the parameters are good for the plant. The correspondence between parts and parameters is shown in Table 2 . Examples of facial pictures are shown in Fig. 4.

Bioelectric potential corresponds to a blink. When a user touches the plant, the potential changes immediately. We use this characteristic in our system. This correspondence in the system enables the interaction between the user and the plant by touch. 
Table 2

Correspondences between environment and facial part.

\begin{tabular}{lc}
\hline Environmental parameter & Corresponding part of the face \\
\hline Temperature & Color of cheeks \\
Humidity & Shape and size of mouth \\
$\mathrm{CO}_{2}$ & Angle of brows \\
Wind velocity & Movement of hair (animation) \\
Light quantum & Size of eyes \\
Moisture & Color of hair \\
Bioelectric potential & Blink \\
\hline
\end{tabular}

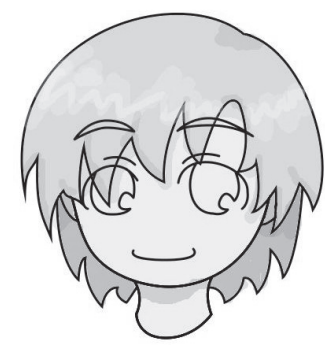

(a)

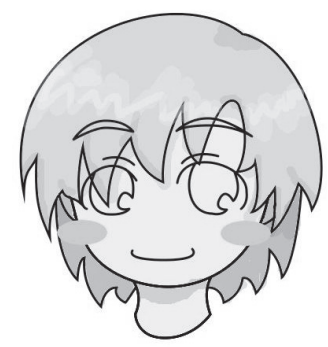

(c)

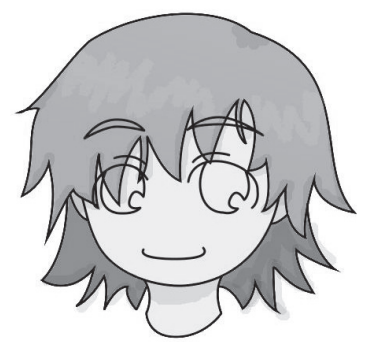

(b)

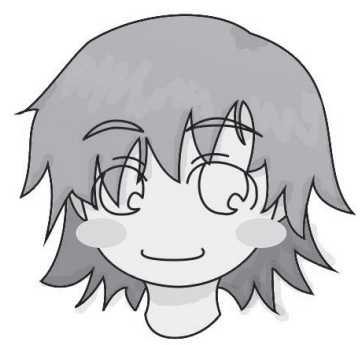

(d)

Fig. 4. Examples of facial pictures. (a) Normal state, (b) low-moisture state (hair color becomes brown), (c) high-temperature state (color of cheeks becomes red) (d) high-temperature and lowmoisture state (mixture of (b) and (c)).

\subsection{Communications and examples}

Using the proposed system, a user can communicate with the plant through the facial picture and voice. The system has a feature of voice recognition and it can reply to a user's question using a voice synthesizer. However, it is difficult to recognize semantics of the user's question; the system cannot reply to all questions. In this study, the system recognizes simple greetings or words in questions about the environment of the plant. For other questions, the system will reply by nodding or back-channeling. 
When the system accepts a question about the environment, for example, "Is it cold?", it replies with a favorable message when the parameter related to the user's question is good for the plant. On the other hand, when the parameter is not good for the plant, the system replies with an unfavorable message and it requests the user to improve the environment. For example, if it is too cold for the plant, it requests the user to close the window or turn on the heater, and so on. Relationships between actions and effects on the environment are stored in the knowledge base of the system. An example of knowledge is shown in Table 3.

The following are examples of communication between the user and a plant.

\section{Example 1}

User: "Good morning."

Plant: "Good morning."

User: "Is it cold?"

Plant: "It is too cold. Please place me under the sunshine."

[Moving the plant near a window.]

[Temperature increases.]

User: "OK. Is it still cold?"

Plant: "It feels good. Thank you very much."

Example 2

User: "Hello"

Plant: "Hello"

User: "Is it dry, today?"

Plant: "I am thirsty. Please give me water."

[Providing water to the plant.]

[Soil moisture content increases.]

User: "Do you need some more water?"

Plant: "It is OK. Thank you."

Table 3

Knowledge base of relations between actions and effects.

\begin{tabular}{lcccccc}
\hline User's Action & \multicolumn{5}{c}{ Change in environment } \\
\cline { 2 - 6 } & Temperature & Humidity & $\mathrm{CO}_{2}$ & Light \\
density & quantum & $\begin{array}{c}\text { Wind } \\
\text { velocity }\end{array}$ & $\begin{array}{c}\text { Moisture } \\
\text { content }\end{array}$ \\
\hline $\begin{array}{l}\text { Opening the } \\
\text { window/door }\end{array}$ & $?$ & $?$ & $\downarrow$ & & $\uparrow$ & \\
$\begin{array}{l}\text { Closing the } \\
\text { window/door }\end{array}$ & $?$ & $?$ & & & $\downarrow$ & \\
$\begin{array}{l}\text { Providing } \\
\text { water }\end{array}$ & $\downarrow$ & $\uparrow$ & & & & $\uparrow$ \\
$\begin{array}{l}\text { Moving to a } \\
\text { sunny place }\end{array}$ & $\uparrow$ & & $\downarrow$ & $\uparrow$ & & $\downarrow$ \\
$\begin{array}{l}\text { Moving to a } \\
\text { shady place }\end{array}$ & $\downarrow$ & & $\uparrow$ & $\downarrow$ & & \\
\hline
\end{tabular}

$\uparrow$ : increase, $\downarrow$ : decrease, ?: unknown (depends on air through open window) 


\section{Experiment and Considerations}

An experiment to examine the applicability of the proposed system to reduce mental stressors is carried out. In the experiment, users communicate with the plant using the proposed system, then, the stress level is measured before and after communication. Nipro's "Cocoro-Meter" is used to measure the stress level. The "Cocoro-Meter" measures amylase activity to easily estimate the stress level in humans. ${ }^{(5)}$

The number of subjects in this study is 15 . They are students of a university. The procedure of this experiment is as follows.

Step 1: Start communication by greeting the plant.

Step 2: Ask the plant about the environment. That is, a subject asks a question about temperature, humidity, moisture, etc. in freely constructed sentences.

Step 3: The proposed system will reply to the subject's question.

Step 4: If the reply contains a complaint about the environment, the subject acts to improve the environment.

Step 5: The system detects a change in the environment, and the plant thanks the subject for improving the environment vocally or through a facial picture.

The subject and plant communicate with each other by repeating Steps 2 to 5 until the environment becomes good for the plant.

In this experiment, a pothos (Scindapsus aureus) plant is used, and its suitable domains of parameters are shown in Table 4. Results of the experiments are shown in Fig. 5. In Fig. 5, when the amylase activity is between 0 and 30, it means no stress is detected in the user, when the amylase activity is between 31 and 45, it means there is slight stress, when the amylase activity is between 46 and 60, it means there is some stress, and when the amylase activity is over 61, it means there is considerable stress. Figure 6 shows the difference in stress level before and after communication. In Fig. 6, a large difference means that stress is reduced to a great degree by the communication.

From the results, the average difference in stress level was -4.53 . The stress level in 5 subjects (Nos. 1-3, 5, 6) was reduced, that is, their stress level changed to a lower level. However, that in 7 subjects (Nos. 7-12, 14) did not change, and the stress level increased in 3 subjects (Nos. 4, 13, 15). The causes of this result are considered to be as follows.

Table 4

Suitable domains of parameters for pothos.

\begin{tabular}{lc}
\hline Environmental parameter & Suitable domain \\
\hline Temperature & $10-26\left({ }^{\circ} \mathrm{C}\right)$ \\
Humidity & $40-60(\%)$ \\
$\mathrm{CO}_{2}$ density & $350-5,000(\mathrm{ppm})$ \\
Wind velocity & $0.15-5.5(\mathrm{~m} / \mathrm{s})$ \\
Light quantum & $300-10,000(\mathrm{~lx})$ \\
Moisture content & $650-1,000(\mathrm{mv})$ \\
\hline
\end{tabular}




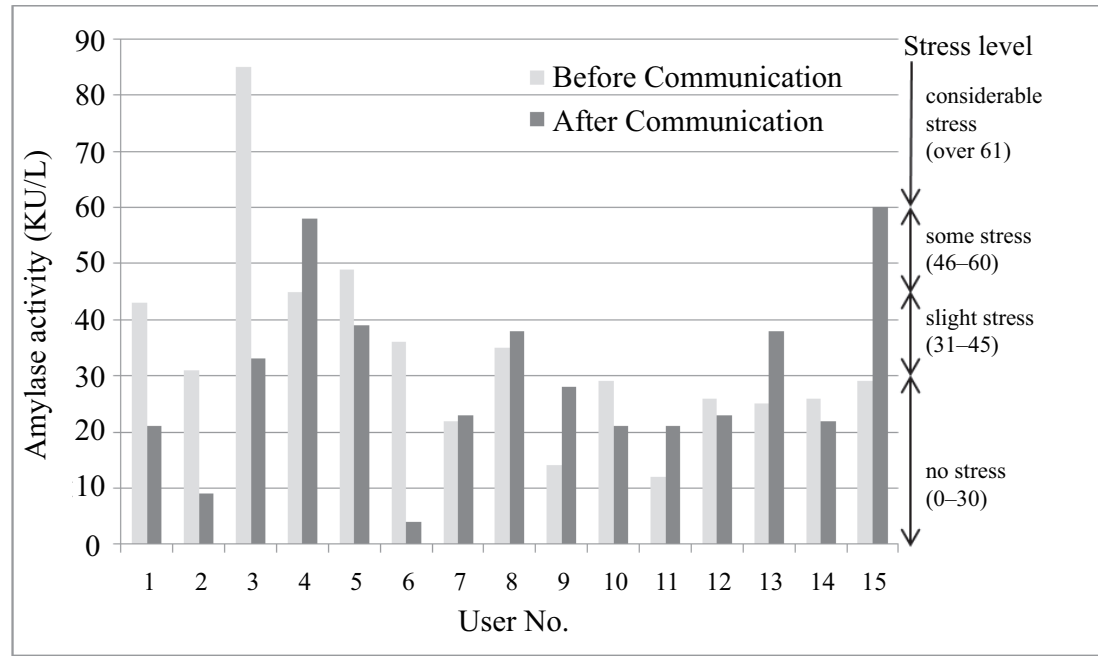

Fig. 5. Results of communication experiment.

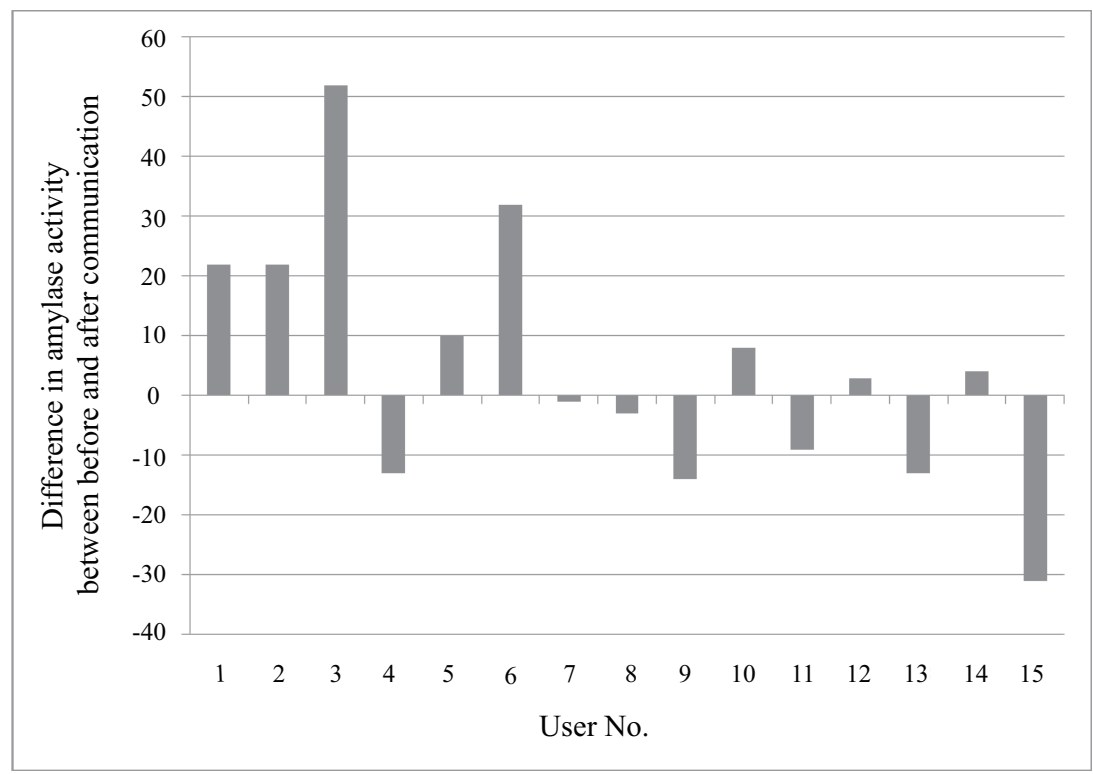

Fig. 6. Stress level difference between before and after communication. 
First, it is considered that the subject's action sometimes does not affect the environment of the plant. For example, the system requires an improvement of wind velocity by opening the window; however, no such improvement occurs when the subject opens the window. Therefore, the subject is not thanked by the plant and a satisfactory communication is not established. Secondly, when the environment is already suitable for the plant, the communication between the subject and the plant is over after only the greetings. It is not effective to reduce stressors. Additionally, the failure to recognize the voice may stress the subject.

Considering from another viewpoint, we pay attention to the subject with a high stress level before the communication. Figure 7 shows differences in stress level determined from the user's stress level before communication. As shown in Fig. 7, we can find the tendency that the reduction of stress level is larger when the stress level before communication is higher. Therefore, our proposed system has some healing effect on a person feeling mental stressors.

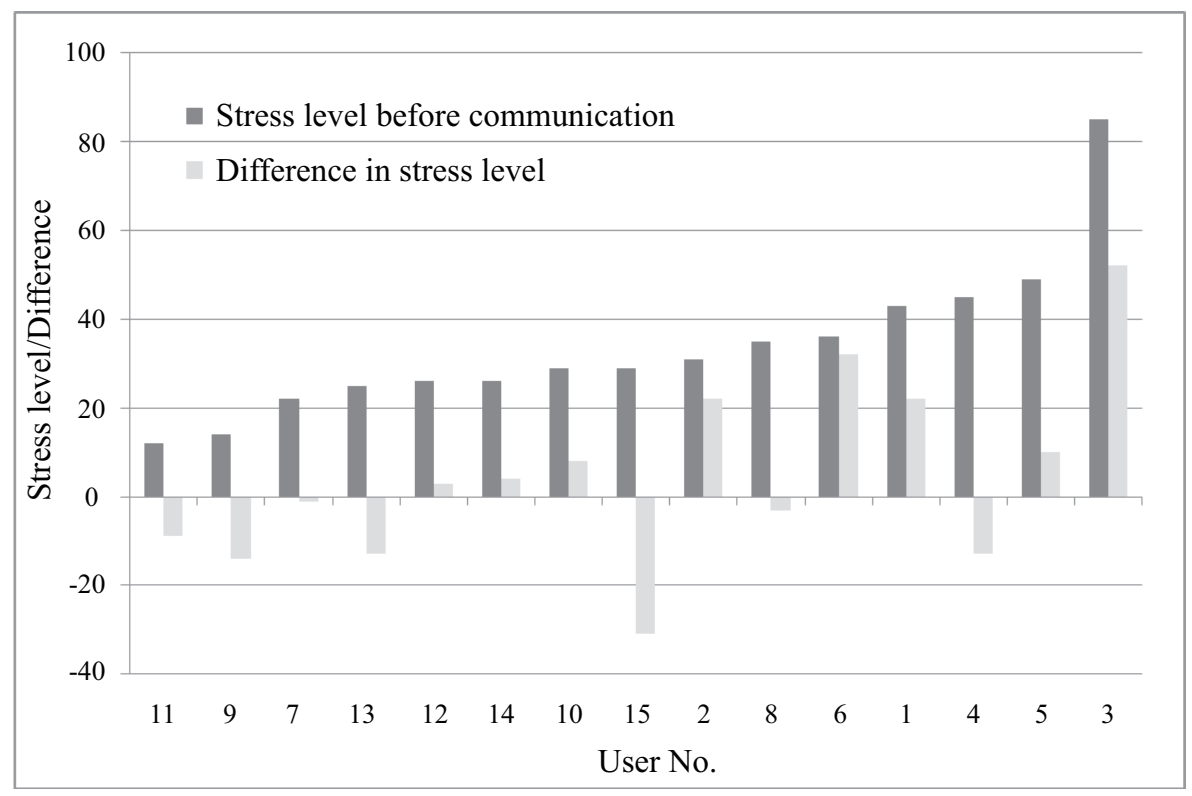

Fig. 7 Stress level before communication and its difference. 


\section{Conclusions}

In this paper, we propose a system of communication with plants to reduce the mental stress of users. The communication system is realized using a plant and multisensors, and users can communicate with the plant through a microphone, display, and tactile sensations.

Then, from the amylase activity of a user under mental stress, it was observed that the stress level decreases after using the system. Therefore, it is clear that our system reduces mental stress to some extent.

In our future work, we will add more communication patterns to the system to cover more situations. Also, the accuracy of voice recognition needs to be improved. These points are important for the system to be more effective against mental stress.

\section{References}

1 Health Technology Research Center: http://unit.aist.go.jp/htrc/team/ment.html.

2 Y. Hasegawa, H. Uchida, S. Asada, T. Katsube and T. Oyabu: The Society of Plant Engineers Japan 14 (2002) 96.

3 X. Zhao, T. Sugawara, S. Kuroda, J. Arisawa and K. Kimura: IEICE Technical Report ME and Biocybernetics 106 (2006) 21.

4 T. Shimbo, T. Oyabu, S. Hirobayashi and K. Takenaka: Transaction of IEE of Japan 124-E (2004) 470 .

5 M. Yamaguchi, T. Kanemori, M. Kanemaru, Y. Mizuno and H. Yoshida: Sensors and Materials 15 (2003) 283.

\section{About the Authors}

Hidetaka Nambo received his B.E. degree in 1994 from the Department of Electrical Engineering and Information, Kanazawa University, and his M.E and Ph.D. degrees in 1996 and 1999, respectively, from Kanazawa University. He was a Research Associate in 1999 in the Department of Electrical and Information, Faculty of Engineering, Kanazawa University. Since 2001, he is a lecturer in the Graduate School of Natural Science and Technology, Kanazawa University. He is engaged in research on the user authentication algorithm of pet robots and data mining. He is a member of the IEE of Japan, the Institute of Electronics, Information and Communication Engineers (IEICE) of Japan, IPSJ of Japan, and IEEE.

Taiki Nakagawa graduated from the Department of Information and System Engineering, Kanazawa University, Japan in 2009. Currently, he is in the master course in the Division of Electrical and Computer Engineering. He is interested in developing a system for interaction with plants. 
Haruo Maegawa graduated from the Department of Information and Systems Engineering, Faculty of Engineering, Kanazawa University, Japan in 2009. He is majoring in Electrical and Computer Engineering in the Graduate School of Nature Science and Technology of Kanazawa University. He studies combining plant states and facial images to communicate with plants.

Haruhiko Kimura graduated in 1974 from the Engineering Department of Tokyo Denki University specializing in Applied Physics and Chemistry. In 1979, he completed his doctoral course in Information Engineering in the Graduate School of Engineering at Tohoku University. He holds a $\mathrm{PhD}$ in engineering. Also, in 1979, he joined Fujitsu Corporation. In 1980, he became a lecturer in Kanazawa Women's Junior College. In 1984, he became an assistant professor in the Economics Department of Kanazawa University. Currently, he is a professor in the Information Systems Engineering Department of the Engineering Faculty of Kanazawa University. At this time, he is engaged in research on optimal code conversion and the acceleration of production systems. He is a member of the IEICE, the Japanese Society for Artificial Intelligence, and the Information Processing Society of Japan. 